\title{
Feasibility and Advantages of a Modified Subxiphoidal Video-Assisted Thoracoscopy Procedure Compared to Established Traditional Video-Assisted Thoracoscopy Procedures in Intercostal Access
}

\author{
Mohammed Ibrahim, $M D^{1^{*}}$ (D) Bernd Linsmeier, $M D^{1}$ and Matthias Steinert, $M D^{2}$ \\ ${ }^{1}$ Department of Thoracic Surgery, Bamberg Sozialstiftung Hospital, Germany \\ ${ }^{2}$ Department of General, Visceral, Transplantation, Thoracic, and Vascular Surgery, University Hospital of Leipzig, Germany
}

*Corresponding author: Mohammed Ibrahim, MD, Department of Thoracic Surgery, Bamberg Sozialstiftung Hospital, Buger Str. 80, 96049, Bamberg, Germany

\begin{abstract}
Background: Minimally invasive thoracic surgical techniques are under continual development, especially the subxiphoidal approach, which until recently could only be done in a few centers across the world, due mainly to the many challenges associated with this method. There are ongoing discussions around minimally invasive methods including the instrument sizes and types, the incision position and expected outcomes. Robotic surgery using the subxiphoidal approach is still under discussion and is expected to be one of the methods of the future.

In this study we aim to demonstrate the advantages of our subxiphoidal technique using a modified incision position and size, combined with a separate 5-10 $\mathrm{mm}$ camera incision in the 6-7 midaxillary line.

Methods: This is a retrospective review of our clinical data obtained from modified subxiphoid procedures performed between July 2018 and March 2020. In total, 50 cases (30 Female, and 20 Male) were identified in which our modified subxiphoid video-assisted thoracoscopic surgery (VATS) was applied including cases of lobectomy, segmentectomy, wedge resection, hemothorax, chylothorax, and biopsies from mediastinal tumors and lymph nodes. Institutional Review Board (IRB) and ethics commission approval of our study have been given by the University of Erlangen and patient consent gained.
\end{abstract}

Results: 50 modified subxiphoid VATS procedures were performed between July 2018 March 2020, including lobectomy, segmentectomy such as apical tri-segmentectomy of the left upper lobe, the basal segmentectomy of both sides, wedge resection, hemothorax, chylothorax, and biopsies from mediastinal tumors and lymph nodes. There were no mortalities, no complications of postoperative bleeding, one case of surgical chylothorax, and three cases of parenchymal air leakage which were treated with pleurodesis, and postoperative arrhythmia.

Conclusions: The modified subxiphoid incision, combined with a separate camera incision showed many advantages for the patients, such as a smaller subxiphoid incision even for lobectomy $(3.5 \mathrm{~cm})$, less pain, more space in which to apply the instruments in situ, accurate view of the posterior mediastinum for the completion of subcarinal lymph node dissection and the dissection of adhesions. No pain was reported by patients at the position of the camera incision, no abdominal muscle layer weakness or herniation, shorter recovery and stay in hospital and no wound disorders.

\section{Keywords}

Minimal invasive thoracic surgery, Modified subxiphoidal VATS access
Abbreviations
CT: Computer Tomography; GGO: Glass Ground Opacity; VATS: Video Assisted Thoracoscopy; EBUS: Endobronchi- al Ultrasound; PET: Positron Emission Tomography; MR: Magnetic Resonance; BMI: Body Mass Index

Citation: Ibrahim M, Linsmeier B, Steinert M (2021) Feasibility and Advantages of a Modified Subxiphoidal Video-Assisted Thoracoscopy Procedure Compared to Established Traditional Video-Assisted Thoracoscopy Procedures in Intercostal Access. Int J Surg Res Pract 8:127. doi.org/10.23937/23783397/1410127

Accepted: March 26, 2021; Published: March 28, 2021

Copyright: (c) $2021 \mathrm{Ibrahim} \mathrm{M}$, et al. This is an open-access article distributed under the terms of the Creative Commons Attribution License, which permits unrestricted use, distribution, and reproduction in any medium, provided the original author and source are credited. 


\section{Introduction}

Recently, there has been a huge development in minimal Invasive thoracic surgical methods worldwide. These include uni-, duo-, triportal video-assisted thoracoscopic surgery (VATS), and subxiphoid robotic surgery techniques. These methods have shown clear positive results in reduced recovery periods for patients, reduced pain score, reduced wound disorders, and improved aesthetics [1-16].

However, subxiphoidal VATS procedures are a modern modification of VATS, which are only performed at a few specialized centers. These procedures and their related results are still undergoing scientific evaluation $[2,5-8,13,15-17]$.

Therefore, we decided to modify our subxiphoid VATS operational technique and evaluate the safety, efficiency, challenges and feasibility of this surgical procedure.

Here we report on the establishment of a modified subxiphoidal approach, indications and contraindications as well as its advantages in terms of a significant reduction in tissue traumatization, reduced drainage duration, faster convalescence, reduced use of painkillers, shorter hospital recovery, and maintenance of the xiphoid process for its benefits and anatomical functions.

Nardini, et al. reported using multiple small accesses including the subxiphoid approach in microlobectomy in July 2018 [18].

\section{Materials, Indications and Methods}

We retrospectively analyzed the results of 50 pa- tients who underwent modified duo-portal subxiphoidal VATS procedures, which included 10 lung wedge resections, as lunge volume reduction or pneumothorax, 10 diagnostic of focal ground-glass opacity pulmonary nodules (GGO), 2 mediastinal lymphadenectomy for histological items, 3 hemothoraces and chylothoraces, and anatomical resection as 16 lobectomies and 9 segmentectomies with radical Lymphadenectomy (Table 1).

\section{Preoperative preparation}

Medical history and physical examination were undertaken including blood tests, thoracic X-ray in two profiles and lung function. Lung perfusion tests were done for lung volume reduction.

Standard staging was done using fluorodeoxyglucose positron emission tomography/CT (FDG-PET/CT), MRI head and bronchoscopy with endobronchial ultrasound (EBUS) and biopsies for any confirmed malignancy and malignancy suspected pulmonary nodules.

In the oncological cases, histological diagnostics were done through EBUS-transbronchial needle aspiration (TBNA) and were mostly cases of adenocarcinoma, squamous cell carcinoma, typical and atypical carcinoid.

In cases of non histological diagnostic and PET-positive pulmonary nodules without any extrathoracic metastasis, we have done a standard intraoperative frozen section procedure.

The decisive criterion for successful completion of a subxiphoid VATS is the careful selection of patients. In particular, patients with cardiac diseases associated with heart failure and consecutive enlargement of the heart, as well as previous operations on the heart (ad-

Table 1: Type of operation.

\begin{tabular}{|c|c|c|}
\hline Type of operation & Side & Number \\
\hline \multirow{2}{*}{$\begin{array}{l}\text { Lunge wedge resection as lung volume reduction, } \\
\text { pneumothorax }\end{array}$} & Right & \multirow[t]{2}{*}{10} \\
\hline & Left & \\
\hline \multirow{2}{*}{$\begin{array}{l}\text { Lunge wedge resection as GGO or pulmonary nodules, } \\
\text { mediastinal tumors }\end{array}$} & Right & \multirow[t]{2}{*}{10} \\
\hline & Left & \\
\hline \multirow[t]{2}{*}{ Hemothorax, chylothorax } & Right & 1 \\
\hline & Left & 1 \\
\hline \multirow[t]{2}{*}{ Diagnostic mediastinal lymphadenectomy/Biopsies } & Right & 2 \\
\hline & Left & 1 \\
\hline \multirow[t]{5}{*}{ Lobectomy } & Right: Upper lobe & 3 \\
\hline & Middle lobe & 1 \\
\hline & Lower lobe & 3 \\
\hline & Left: Upper lobe & 5 \\
\hline & Lower lobe & 4 \\
\hline \multirow[t]{4}{*}{ Segmentectomy } & Right: Basilar group segmentectomy $(S 7,8,9,10)$ & 2 \\
\hline & $\begin{array}{l}\text { Left: Basilar group segmentectomy } \\
(\mathrm{S} 8,9,10)\end{array}$ & 2 \\
\hline & Tri-apical group segmentectomy left & 1 \\
\hline & Segment S6 & 4 \\
\hline
\end{tabular}




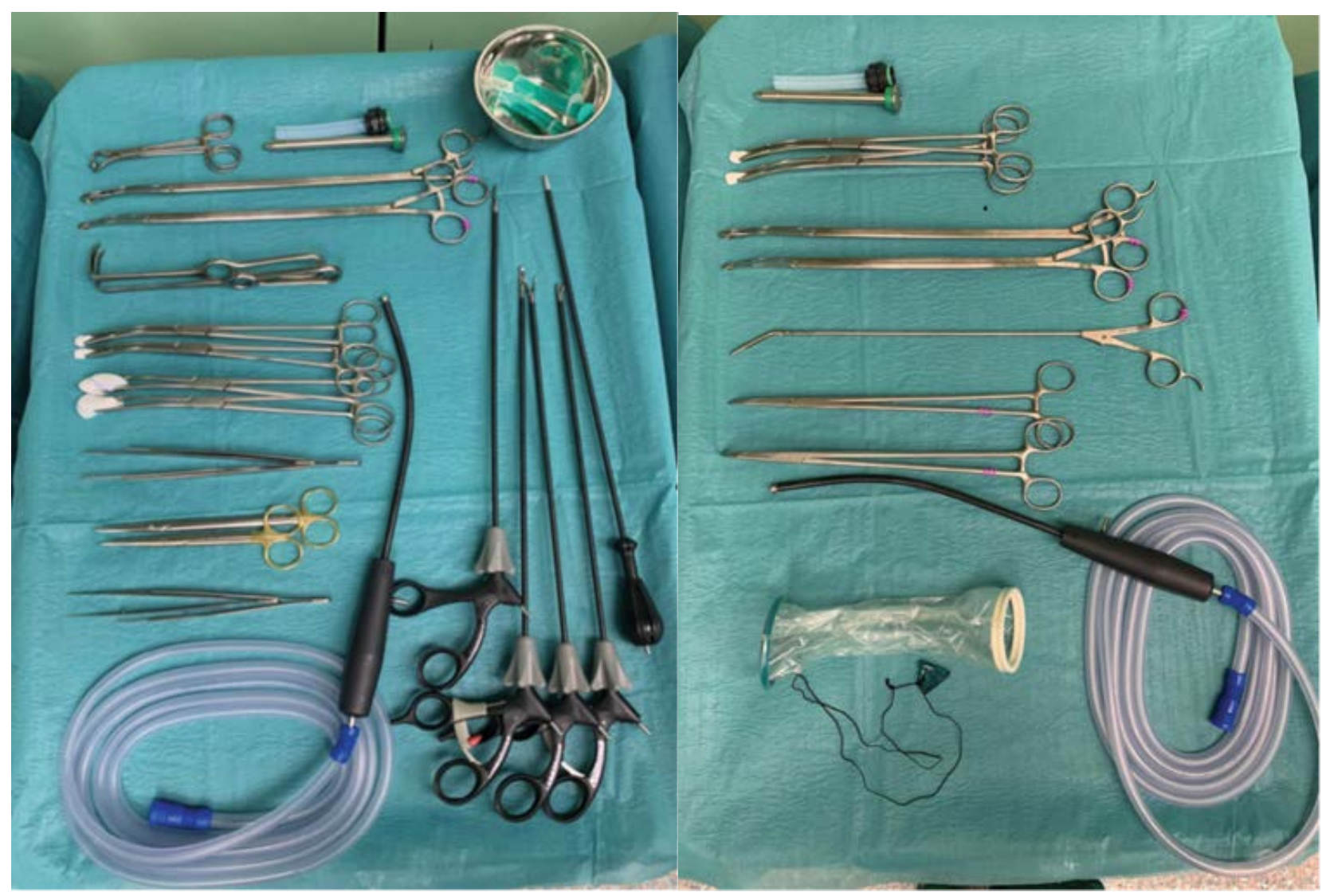

Figure 1: $(A, B)$ The surgical instruments used during the performance of subxiphoid approach, such as duffner/aesculap or scanlan instruments, harmonic for sharp and blunt preparation of the hilar or interlobar structures, suction system, alexis ring protection folie.

hesions) limit the access of a subxiphoidal procedure, particulary on the left side. This is mainly due to issues of confinement and intraoperative manipulation of the instruments on the heart itself, which can trigger blood pressure disorders and cardiac arrhythmia. An appropriate and careful preoperative examination is needed in these cases, including echocardiogram, transthoracic echocardiography, chest X-ray and CT chest with contrast. Another important selection parameter is the obesity index $\left(\mathrm{BMI}>30 \mathrm{~kg} / \mathrm{m}^{2}\right)$. Here, the access depth can be significantly extended by subcutaneous layers which significantly complicates procedure preparation and increases the potential for wound healing disorders. Regarding functional operability, the same exclusion criteria apply as for conventional operations or traditional VATS methodology.

This modified approach requires experienced thoracic surgeons and anaesthetist with a background in uniduo portal VATS procedures.

\section{Operative procedures and instruments}

We performed all the operations under general anesthesia after double-lumen tube intubation, with or without peridural epidural pain catheter.

Surgical Instruments: Figure $1 \mathrm{~A}$ and Figure 1B.

- 5-10 mm camera optic.
- Duffner/Aesculap or Scanlan instruments.

- Harmonic for sharp and blunt preparation of the hilar or interlobar structures.

- Storz and Olympus Monitoring.

- Stapling devices (Ethicon $60 \mathrm{~mm}$ gold, $35 \mathrm{~mm}$ white vascular, $35 \mathrm{~mm}$ green bronchus).

\section{Subxiphoidal modified duo-portal VATS technique}

Position: All our interventions were carried out in a lateral position on a vacuum mattress.

The procedure is performed under general anaesthesia, intubation with double lumentube and anterolateral one-lung ventilation.

The patient is placed in a supine position with $30-40^{\circ}$ retroversion and sometimes with $60-70^{\circ}$. The ipsilateral arm can be positioned, and in our case fixed, above the head of the patient in a holder, taking care of the shoulder position to avoid any damage of the brachial plexus. The Standard Set [2,5-8,13,15-17] (Figure 2).

The standard positioning of the patient, surgeons, assistants and scrub nurse are done as follows; both main surgeons at the front (ventral) of the patient, the assistant and the scrub nurse on the opposite side, and the double monitor tower set in front of the surgeons and assistant. 


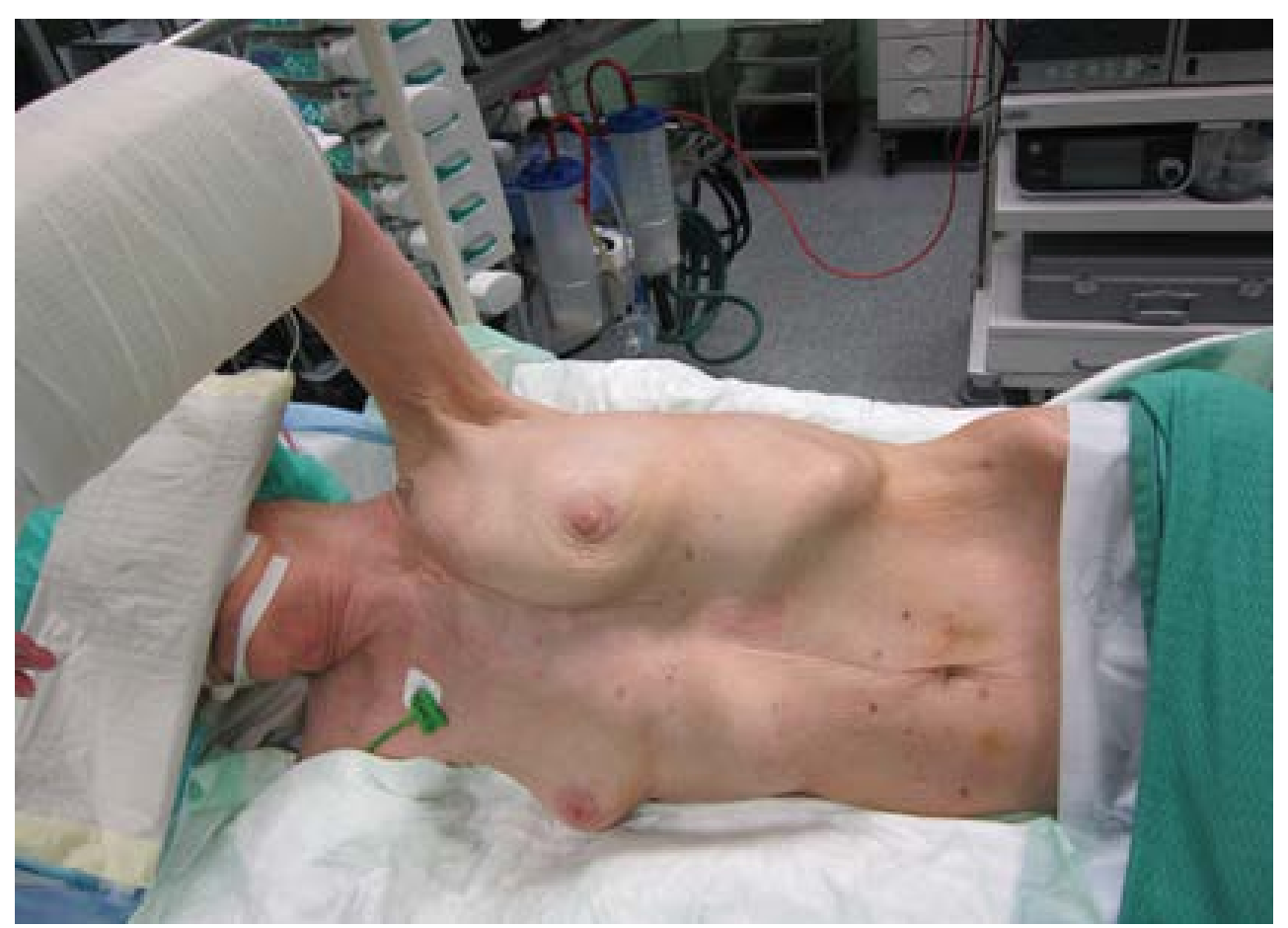

Figure 2: The position of the patient in supine with $30-40^{\circ}$ with the fixation of the ipsilateral arm over the head.
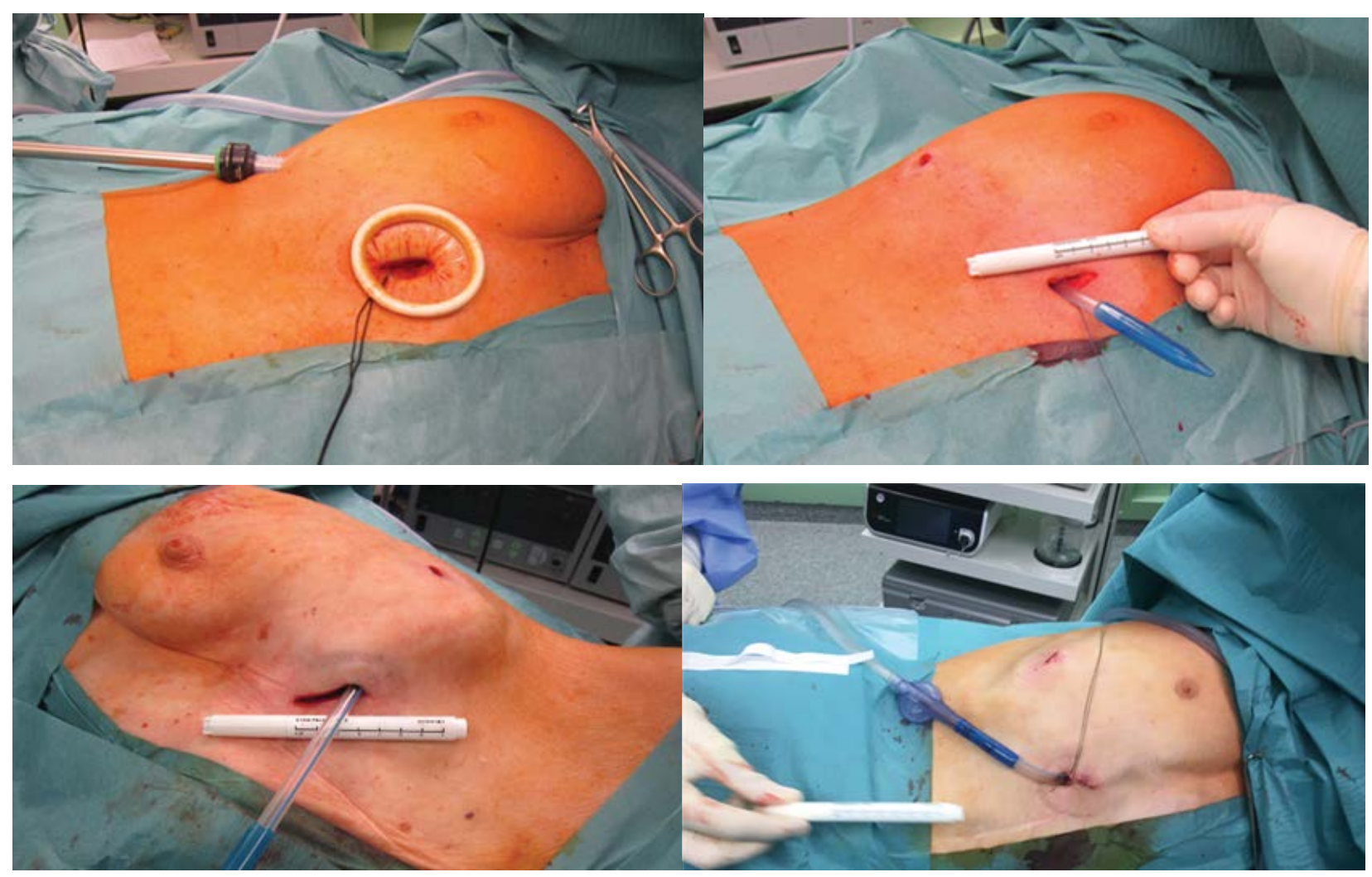

Figure 3: We describe the main and camera optic incisions in the subxiphoid and intercosal postions. (A) The position of subxiphoid incision on the right side during lobectomy of the right upper lobe, with separate incision access for the optic camera; (B) At the end of the operation, the position of the chest tube through the ca. $3 \mathrm{~cm}$ long subxiphoid incision access; (C) At the end of the operation after lobectomy of the left lower lobe, the position of the chest tube through the ca. $3.5 \mathrm{~cm}$ long subxiphoid incision access; (D) U-shaped suture and fixing the chest drain, the layered wound is finally closed with donati-sutures method.

It should also be mentioned that the decision regarding the position of the surgeons according to the patient's position and choice of instruments is different for patients from Western European cultural area comparing with those patients from Asia.
Incision: As a first "crucial step" after the sterile wash and covering of the surgical field, we make a small incision in the 6-7 intercostal space in the posterior-mid axillary line and introduce a trocar for a 5-10 mm camera optic into the pleural space. After an appropriate lung 
atelectasis has been performed, creating an appropriate mediastinal shift the surgical site is identified and the choice of subxiphoidal procedure confirmed. Then the optimal position for the main subxiphoidal access can be decided under direct view of the camera. A slightly oblique (transverse) skin incision $(3-4 \mathrm{~cm})$ is made between the xiphoid process and the medial rib cage. After the skin and subcutaneous tissue have been severed, an incision is made in the anterior rectus abdominis muscle sheath and digital blunt dissection performed under direct vision of the camera to reach the pleura cavity. This procedure avoids the removal of the xiphoid process and massively reduces the trauma of access tissues $[8,15-17]$.

After inserting an XS Alexis O-ring, the operation can proceed. The use of a retractor is not necessary. By sparing the posterior fascia of the rectus abdominis muscle, there is virtually no danger of abdominal herniation or weakness of the abdominal muscles, and bleeding from the epigastric vessels can also be avoided. Thoracic drainage is also achieved via the subxiphoidal approach thus preventing damage to the neurovascular bundle in the intercostal space, which subsequently significantly reduces postoperative pain. The wound closure takes place layer by layer with final intracutaneous suture and hydrocolloid dressing (Comfeel-Plus) in the area of the lateral camera incision. After placing a U-shaped suture and fixing the chest drainage, the layered wound is finally closed with Donati-suture method (Figure 3A, Figure 3B, Figure 3C and Figure 3D).

Some Other experienced thoracic surgeons performed a bilateral subxiphoidal approach for bilateral oncological and non-oncological resection applying a horizontal Incision over the subxiphoidal area, and resection of the xiphoid process to facilitate [5-7], and others keep the xiphoid process and the rectus abdominis was divided at the midline to expose the xiphoid process, localizing find the infrasternal angle to perform the subcutaneous tunnel till reach the pleural cavity [8].

General intraoperative procedures: After applying the subxiphoid incision on the right side under direct vi- sion of the camera and gaining access to the pleura cavity, the presentation and dissection of the hilus including the upper lung vein, middle lobe vein and lower lung veins is straightforward. Furthermore the upper lobar artery (truncus anterior) and of course the interlobium with the pulmonary artery and all of its branches, especially the middle lobe, lower lobe basilar artery, the segment 6 artery and segment 2 artery are easy to reach. Therefore the middle and lower lobectomy showed no great challenges but the upper lobectomy requires long angled (curved) instruments especially for patients from North European Countries who tend to have a larger chest wall, wider pleural cavity, and longer subcutaneous tunnel between the incision and pleural cavity (Figure 4A and Figure 4B).

The approach on the left side showed more challenges than on the right side due to the position of the heart, specifically in the case of a hypertrophic heart, but the presentation and dissection of the hilus, blood vessels of both lobes, was as straightforward as the same procedure carried out on the right side. By carefully selecting the patients and evaluating heart size on the thoracic CT scan, these challenges can be avoided (Figure 5A, Figure 5B, Figure 5C and Figure 5D).

In addition, the subxiphoid approach showed better results and no significant challenges compared with the traditional intercostal approach, with regard to overcoming and removing mediastinal adhesions attributed to previous cardiac operations including sternotomy, pneumonitis and intrapleural cavity diffuse of infectional adhesions. This is due to the more convenient angle (view from down to up) at which the mediastinum, the diaphragm, and the anterior-posterior costophrenic angle are presented $[1,17]$.

We addressed some of the difficulties in localizing deep intraparenchymal GGO nodules, due to the difficulty of digitally palpating it with this technique, but in such cases we would advise marking the pulmonary nodules using radio guided occult lesion localization (ROLL) with technetium-99m macroaggregated albumin (TcMAA)/methylene blue in order to resect the nodules

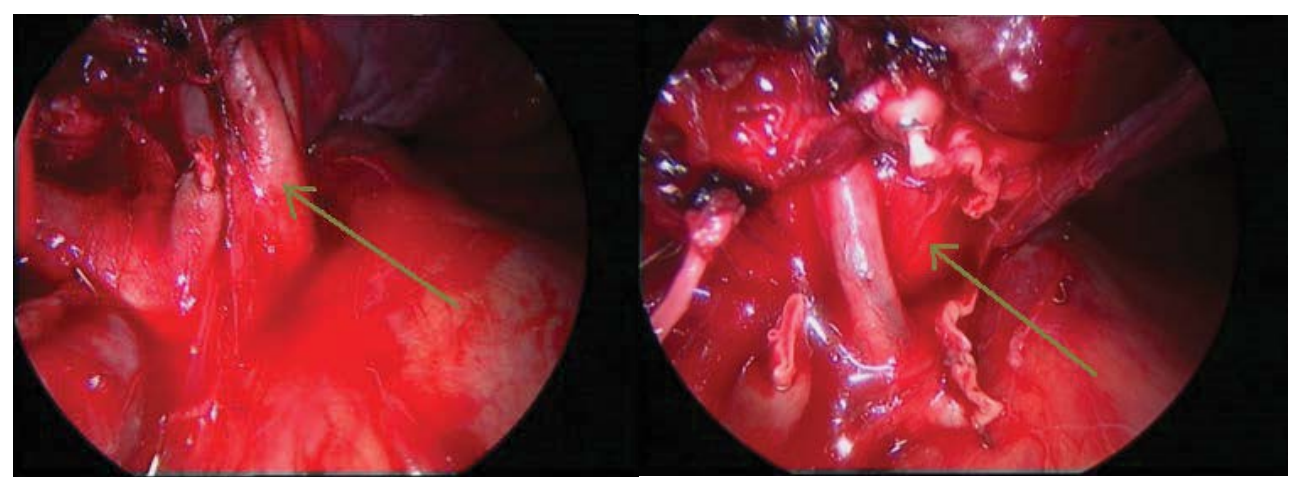

Figure 4: Dissection of the veins and arteries during the lobectomy. A) Presenting and dissection the right upper lobe veins and arteries; B) Presenting the already transected A1, A3 (more branches), the $2^{\text {nd }}$ branch of $A 2$, and the right upper lobe bronchus. 


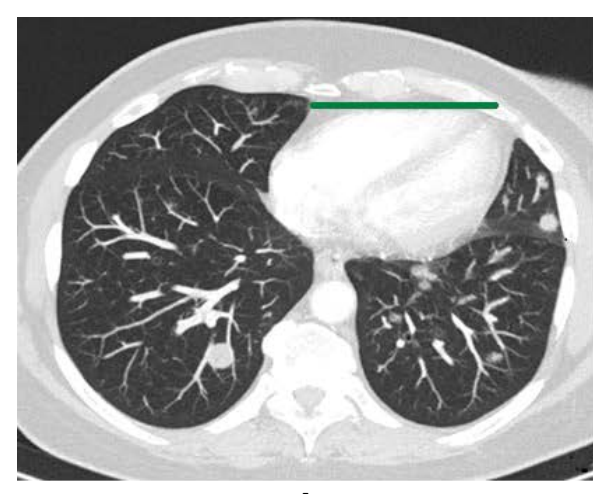

A

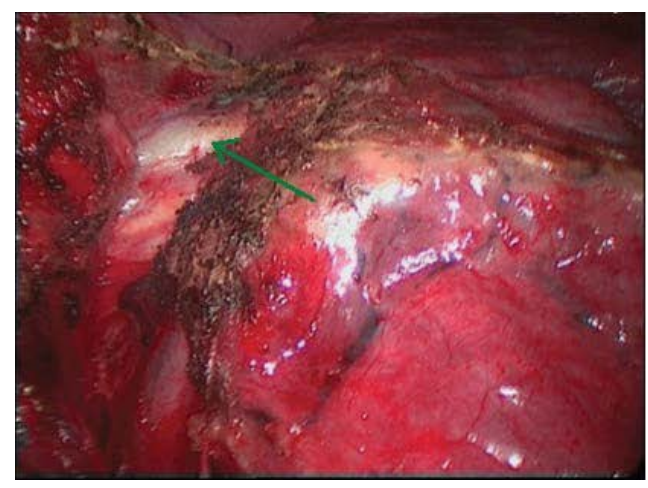

C

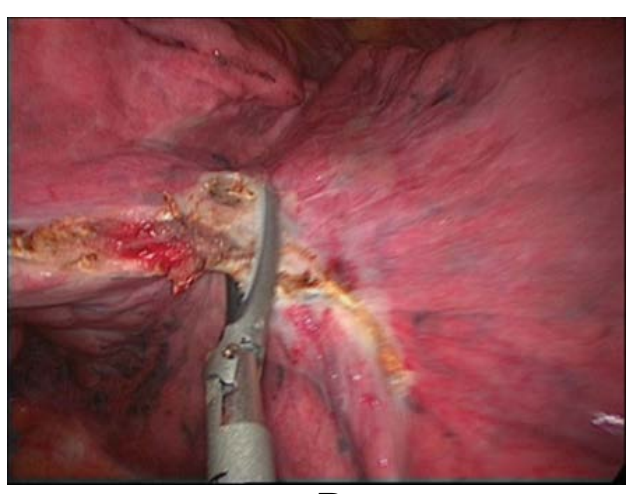

B

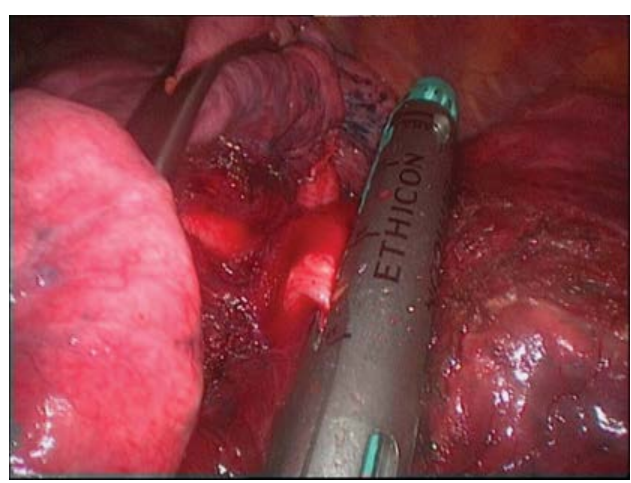

D

Figure 5: Present the CT scan preoperative the clear size of the heart (Cardiomyopathy), dissection of the blood vessels and parenchyma. A) CT scan demonstrates the cardiomyopathy and the contraindicated for performing the subxiphoid approach; B) Transection of the parenchymal tissue between left upper lobe and lower lobe to perform the lobectomy of the left lower lobe; C) Presenting the pulmonary artery in the intelobium left; D) Dissection and transection of the basilar pulmonary artery in the interlobar fissure by performing a lower lobectomy with subxiphoidal approach.

without large loss of lung parenchyma and thus prevent unnecessary thoracotomies [19-21], or perform a traditional intercostal VATS.

\section{Discussion}

Different minimally-invasive procedures have recently been applied in thoracic surgery, as triportal, duo-portal, uniportal VATS and subxiphoidal VATS. Many experienced thoracic surgery centers such as the Pulmonary Hospital in Shanghai, China and other Asiatic, European and American centers have demonstrated the benefits of these procedures, including the Subxiphoidal VATS [1-16].

As these procedures are continually being developed, we successfully modified the subxiphoidal approach as follows:

1. We used a separate camera access incision for a 5-10 mm camera optic in the 6-7 the mid axillary line, in order to give a clear view of the main subxiphoidal access site from the intrathoracic side. This enables the choice of the perfect position for the main access and avoids bleeding from the small vessels in the mediastinal fat tissue and potential phrenic nerve injury.

2. The pleural cavity is clearly presented, including hilus and the posterior mediastinum in order to access lung lesions as well as perform complete resections, subcarinal lymph node dissection, and facilitates the resolution of adhesions especially in the mediastinal position.

3. Optimizing the incision at a slightly oblique (transverse) angle to the skin, incision ca. $3-4 \mathrm{~cm}$ between the xiphoid process and the medial rib cage.

4. Avoiding the removal of the xiphoid process and massively reducing the trauma of access tissues, and maintaining the anatomical insertion and function of the rectus abdominis muscle.

5. Avoiding abdominal hernia and weakness of the abdominal muscles by applying the incision of the anterior rectus abdominis muscle layer laterally, enabling digital blunt and sharp dissection with monopolar cutter under direct camera vision until the pleural cavity has been reached.

6. Avoiding the use of sternal and subcostal retractors, thus further reducing both tissue trauma and pain.

The subxiphoid access is more difficult with many challenges compared with the traditional VATS access due to the longer subcutaneous tunnel, longer distance to the upper and lower hilus of the lung, smaller opera- 
tive angle and mutual interference of more instruments [17]. In order to find a solution with regards to this problem we decided to make the small separate $5-10 \mathrm{~mm}$ camera port-incision.

In relation to the longer distance to upper and lower lung hilus, we optimized the subxiphoidal access using the camera as described above to localize the most accessible subxiphoidal incision for both lung hilus minimizing the tissue trauma, reducing pressure on the heart especially in the left side, and reducing the tendency of bleeding during the dissection and transection of blood vessels.

In addition, we noticed that the distance between the subxiphoid access and lung hilus depends on the epidemiological and geographical population distribution. For example the Asiatic population is completely different in body stature (BMI) and in particular the size, shape and form of the entire thorax. Our experiences in populations from western and northern Europe identified that the subxiphoid approach was more challenging because of:

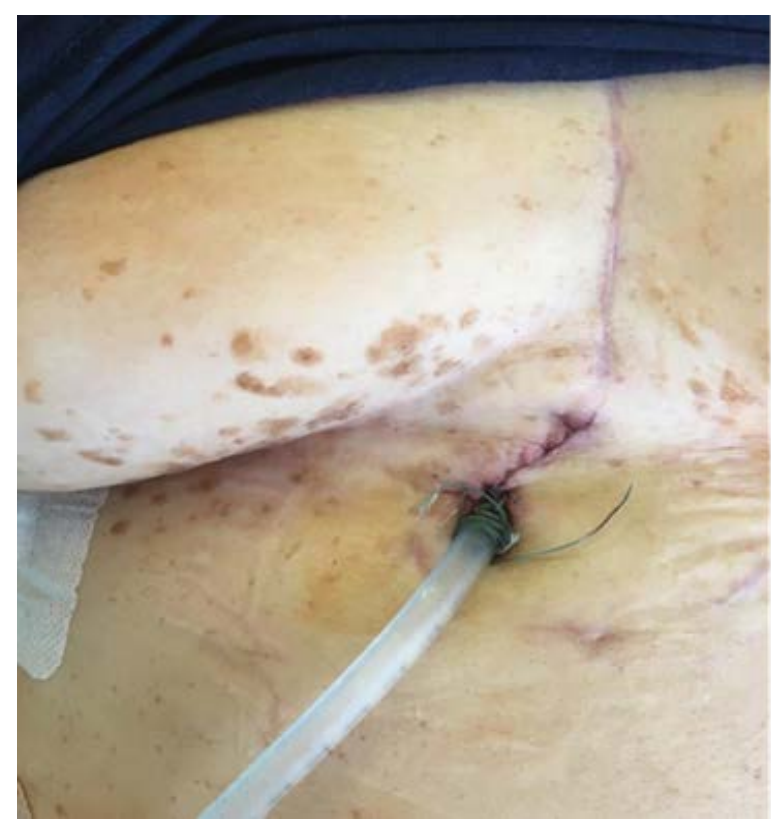

Figure 6: Patient has previously undergone heart surgery via sternotomy. Now the subxiphoidal duo-portal approach was used with right upper lobectomy and systematic lymphadenectomy. Addressing without problem the challenge of surgery with mediastinal adhesions.
1. The thicker subcutaneous layer,

2. The longer distance to hilus of lung,

3. And obesity $(\mathrm{BMI}<25)$.

The position of the subxiphoid incision, very close to the diaphragm and the costophrenic angle, makes for a more convenient presentation of the mediastinal situs, and solves any potential difficulties arising from adhesions of diaphragm and anterior-posterior costophrenic angle compared with that of intercostal uniportal incision. Thus, pleural adhesion was not a contraindication for the subxiphoid uniportal incision procedure $[1,17]$ Figure 6.

The intraoperative bleeding from the rupture of main vessels is harder to control through the subxiphoidal approach compared with the traditional VATS, so in such case we advise compression of the site of bleeding and conversion to thoracotomy to gain access to stop bleeding $[9,17]$.

Special attention must be paid in the choice of instruments for this procedure, and long, articulating endoscopic instruments as produced by Scanlan Inc. (USA) or Duffner Inc. (Germany) are particularly helpful especially in long thoraces.

When modifying our subxiphoid technique we took into consideration other surgical approaches such as the subcostal approach. However this approach still requires cutting the whole external oblique muscle. Moreover, intercostal nerve branches attached to the subcostal area can be affected, which may induce neuralgia and pain [17].

The anterior branches of the intercostal and subcostal nerves supply sensation to the midline abdomen.

We can confirm that we used the same optimized procedures in both the subxiphoid and traditional VATS. There is however, even more capability with the modified subxiphoidal approach with regard to the exposure of the hilus structure, the dissection and the use of endostaplers for transecting the bronchovascular structures and the lung parenchyma. This is due to the comfortable subxiphoidal access angle to expose the above mentioned anatomical positions $[2,5-8,13,15-17]$.

Our experiences demonstrate that anatomical re-

Table 2: Perioperative complications.

\begin{tabular}{|l|l|l|}
\hline Complications & $\begin{array}{l}\text { Subxiphoidal VATS } \\
\text { (n= 50) }\end{array}$ & $\begin{array}{l}\text { Traditional VATS } \\
\text { (n= 300) }\end{array}$ \\
\hline Lung parenchymal air leakage & 3 & 7 \\
\hline Pneumonitis/Emphyema & 0 & 1 \\
\hline Hemothorax & 0 & 1 \\
\hline Chylothorax (with conservative further therapy) & 1 & 1 \\
\hline Cardiological symptoms: Arrythmia & 1 & 3 \\
\hline Postoperative bleeding & 0 & 0 \\
\hline
\end{tabular}


section of every lobe is possible. Even segmentectomy, including apical trisegmentectomy of the left upper lobe, is operable through the modified duo-portal subxiphoidal approach. The lymphadenectomy procedure was more challenging through the subxiphoid approach than by traditional VATS procedure, but thankfully because of the separate camera portal incision we were able to achieve the same number of dissected lymph nodes as in the traditional VATS technique, especially from the subcarinal position. Once optimized and practised the modified approach is easy to apply.

Comparison of postoperative pain between the subxiphoid VATS and traditional VATS has been discussed in different studies. We demonstrated the advantages of the modified subxiphoidal VATS with separate camera incision in that patients complained less about pain, with less need for pain medications, shorter recovery and hospital stay and quicker removal of the chest tube (Table 2).

\section{Conclusion}

The subxiphoid VATS approach is expected to be the next modern approach in thoracic surgery, especially for future robotic procedures (RATS). The modified duo-portal subxiphoid with separate $5-10 \mathrm{~mm}$ camera incision showed advantages for the patients: Demonstrating minimal to no pain at the incision site, the ability to perform different types of operations minimizing the subxiphoid incision with less challenges in lobectomy, wedge resection, mediastinal tumors, lymph node biopsies, lung volume reduction and therapy of the hemothorax, chylothorax.

The careful selection of patients for this approach and the choice of appropriate surgical instruments are both important in order to minimize intraoperative challenges and to achieve great results without complications. Also, performing subxiphoid VATS lobectomies requires a surgeon with previous experience in intercostal VATS lobectomies and a skilled assistant.

Our modified approach clearly demonstrated less postoperative pain comparing with the traditional intercostal VATS approach $[2,6-8,17]$.

Recently, robotic VATS procedures have been applied in thoracic surgery centres worldwide. In Germany, in Jena, Charite' and Leipzig University Hospitals, there have been many oncological resections as lobectomies, segmentectomies, thymectomies using robotic VATS techniques with successful outcomes [11]. So, as robotic VATS surgery is the future of thoracic surgery, then we must consider the possibility of a robotic subxiphoidal approach for oncological resection as lobectomies since the thymectomies have already been done in Germany [14].

We are analyzing the postoperative pain using the numeric pain rating scale. The mean NPRS scores at 1 day, 2 days, 3 days, 4 days, 5 days, and 21 days after surgery will be calculated and further analyzed, respectively for the subxiphoid duo-portal VATS category and for the tri-portal VATS category. So we found out that the postoperative pain were significantly lower in the subxiphoid du-portal VATS patients than in the tri-port VATS. So we will demonstrate and confirm the advantage of subxiphoid duo-portal VATS approach after we will reach 100 cases and will demonstrate the statistical Values and scores [22-28].

\section{Acknowledgements}

None.

\section{Conflict of Interest}

The authors declare that there is NO conflict of interest.

\section{Ethical Approval and Consent to Participate}

The IRB approval of our institute and the Ethick commission approval under number (178_20 Bc) from the University of Erlangen have been given on 25.05.2020 and the consense of the patients includes in this study has been approved and given too before each therapy and operation.

\section{References}

1. Watanabe A, Yamauchi A, Sakata J, Abe T (2001) Extrapleural resection of lung metastasis in a patient with dense pleural adhesions using VATS. Ann Thorac Surg 71: 2015-2016.

2. Chia Chuan L, Chih Shiun S, Yun Hen L, Chih Tao C, Enrico $M$, et al. (2016) Subxiphoid single-port video-assisted thoracoscopic surgery. J Vis Surg 2: 112.

3. Rivas DG, Torre M, Fernandez R, Mosquera VX (2011) Single-port video-assisted thoracoscopic left upper lobectomy. Interact Cardiovasc Thorac Surg 13: 539-541.

4. Rivas DG, Paradela M, Fernandez R, Delgado M, Fieira E, et al. (2013) Uniportal video-assisted thoracoscopic lobectomy: Two years of experience. Ann Thorac Surg 95: 426432.

5. Aresu G, Weaver H, Wu L, Lin L, Sponga S, et al. (2016) Uniportal subxiphoid video-assisted thoracoscopic bilateral segmentectomy for synchronous bilateral lung adenocarcinomas. J Vis Surg 2: 170.

6. Aresu G, Weaver H, Wu L, Lin L, Jiang G, et al. (2016) The Shanghai Pulmonary Hospital uniportal subxiphoid approach for lung segmentectomies. J Vis Surg 2: 172.

7. Arenas LAH, Lei Lin, Yang Y, Liu M, Guido W, et al. (2016) Initial experience in uniportal subxiphoid video-assisted thoracoscopic surgery for major lung resections. Eur J Cardiothorac Surg 50: 1060-1066.

8. Liu CY, Lin CS, Liu CC (2015) Subxiphoid single-incision thoracoscopic surgery for bilateral primary spontaneous pneumothorax. Wideochir Inne Tech Maloinwazyjne 10: 125-128.

9. Grallert M, Uhlmann D, Bartels M, Steinert M (2013) VATS lobectomy-a standard procedure in the therapy for stage I non-small cell lung cancer. Zentralbl Chir 138: S40-S44.

10. Onaitis MW, Petersen RP, Balderson SS, Toloza E, Burfeind WR, et al. (2006) Thoracoscopic lobectomy is a safe 
and versatile procedure: Experience with 500 consecutive patients. Ann Surg 244: 420-425.

11. Petersen R (2016) Video-assisted thoracoscopic thymectomy using 5-mm ports and carbon dioxide insufflation. Ann Cardiothorac Surg 5: 51-55.

12. Sandhaus T, Bönsch T, Grallert M, Doenst T, Steinert M (2017) VATS-Lobectomy: Ready for routine use in stage 1 and 2 non-small cell lung cancer? Thorac cardiovasc Surg 65: S1-S110.

13. Suda T, Ashikari S, Tochii S, Sugimura H, Hattori Y (2014) Single-incision subxiphoid approach for bilateral metastasectomy. Ann Thorac Surg 97: 718-719.

14. Möller T, Egberts J-H, Eichhorn M, Hofmann H-S, Krüger I, et al. (2019) Current status and evolution of robotic-assisted thoracic surgery in Germany-results from a nationwide survey. J Thorac Dis 11: 4807-4815.

15. Wang BY, Chang YC, Chang YC, Wang KM, Lin $\mathrm{CH}$, et al. (2016) Thoracoscopic surgery via a single-incision subxiphoid approach is associated with less postoperative pain than single-incision transthoracic or three-incision transthoracic approaches for spontaneous pneumothorax. J Thorac Dis 8: S272-S278.

16. Yang X, Wang L, Zhang C, Zhao D, Lu Y, et al. (2019) The feasibility and advantages of subxiphoid uniportal video-assisted thoracoscopic surgery in pulmonary lobectomy. World J Surg 43: 1841-1849.

17. Song N, Zhao DP, Jiang L, Bao Y, Jiang GN, et al. (2016) Subxiphoid uniportal video-assisted thoracoscopic surgery (VATS) for lobectomy: A report of 105 cases. J Thorac Dis 8: S251-S257.

18. Marco N, Rocco B, Piergiorgio S, Shruti J, lan Paul, et al. (2018) Microlobectomy: Completely portal pulmonary lobectomy. JOVS 4: 153.

19. Kawada M, Okubo T, Poudel S, Suzuki Y, Kawarada Y, et al. (2013) A new marking technique for peripheral lung nodules avoiding pleural puncture: The intrathoracic stamping method. Interact Cardiovasc Thorac Surg 16: 381-383.
20. Congedo MT, lezzi R, Nachira D, Larici AR, Chiappetta M, et al. (2019) Uniportal VATS coil-assisted resections for GGOs. J Oncol.

21. Mun M, Matsuura $Y$, Nakao M, Ichinose J, Nakagawa K, et al. (2016) Noninvasive computed tomography-guided marking technique for peripheral pulmonary nodules. J Thorac Dis 8: S672-S676.

22. Liu CY, Cheng CT, Wang BY, Shih CH, Liu CC (2015) Number of retrieved lymph nodes and postoperative pain in single-incision and multiple-incision thoracoscopic surgery. Ann Surg 265: e76-e77.

23. Li Y, Wang J, Yang F, Liu J, Li J, et al. (2012) Indications for conversion of thoracoscopic to open thoracotomy in video-assisted thoracoscopic lobectomy. ANZ J Surg 82: 245250.

24. Paul S, Altorki NK, Sheng S, Lee PC, Harpole DH, et al. (2010) Thoracoscopic lobectomy is associated with lower morbidity than open lobectomy: A propensity-matched analysis from the STS database. J Thorac Cardiovasc Surg 139: 366-378.

25. Reinersman JM, Passera E, Rocco G (2016) Overview of uniportal video-assisted thoracic surgery (VATS): Past and present. Ann Cardiothorac Surg 5: 112-117.

26. Wang BY, Tu CC, Liu CY, Shih CS, Liu CC (2013) Single-incision thoracoscopic lobectomy and segmentectomy with radical lymph node dissection. Ann Thorac Surg 96: 977-982.

27. Yim AP (1995) Minimizing chest-wall trauma in video-assisted thoracic surgery. J Thorac Cardiovasc Surg 109: 1255-1256.

28. Waël CH, Moira de V, Eshetu GA, Marcelo C, Thomas KW, et al. (2013) Is video-assisted lobectomy for non-small-cell lung cancer oncologically equivalent to open lobectomy? Eur J Cardiothorac Surg 43: 1121-1125. 